\title{
Systems Biology Approaches-based Biomarkers Discovery for Acute Aortic Dissection
}

\author{
Qiang Huang, MD, Yongqiang Ren, MD, Hui Li, MD, Youjin Qiao, MD, Mingshan Lin, MD \\ Department of Cardiovascular Surgery, Qingdao Municipal Hospital, Qingdao, P.R., China
}

\section{ABSTRACT}

Acute aortic dissection (AAD) faces great challenges in early diagnosis and effective drug treatment. Recent developments in systems biology approaches allow high-throughput screening of novel diagnostic biomarkers and potential therapeutic targets. In this review, we summarize the currently available $\mathrm{AAD}$ biomarkers identified in the context of genomic, transcriptomic, proteomic, and metabolic profiles, and highlight the benefits of using a combination of these findings for a better understanding of the molecular nature of this life-threatening disease. This review also provides a reference for future studies that employ a comprehensive, multiple-level approach at the single-cell level to decipher the underlying molecular pathophysiology of AAD.

\section{INTRODUCTION}

Acute aortic dissection (AAD) is a life-threatening condition characterized by the rapid development of intimal flap and adventitia apart. AAD is most common in those from 65 to 75 years of age. Women are less frequently affected ( 7.9 per 100 $000)$ than men (16 per 100000$)$, but tend to yield worse outcomes [Nienaber 2015]. The acute stage refers to the first two weeks after the onset of $\mathrm{AAD}$, during which patients are most vulnerable to death. The Stanford and DeBakey types are the two most widely used classification systems for AAD. Stanford type A (corresponding to DeBakey type I and II) involves the ascending aorta, and conventionally requires swift open surgical repair. Stanford type B (corresponding to DeBakey type IIIa and IIIb) involves the descending aorta, and is usually treated using endo-vascular repair therapy [Nienaber 2016]. Hypertension and atherosclerosis are the most prevalent risk factors for $\mathrm{AAD}$, and present in up to $75-80 \%$, and $31 \%$ of cases, respectively [Nienaber 2016; Nienaber 2015]. The most common disorders that contribute to AAD include vascular inflammation caused by autoimmune issues or infection, deceleration trauma, and aortic surgery or instrument intervention [Nienaber 2016; Nienaber 2015].

Received March 12, 2018; accepted fuly 2, 2018.

Correspondence: Dr. Mingshan Lin Department of Cardiovascular Surgery, Qingdao Municipal Hospital, Qingdao 266011, P.R.China; (e-mail: qdsslyybuangqiang@163.com).
The rapid diagnosis of $\mathrm{AAD}$ poses a clinical challenge: a missed or delayed diagnosis occurs in $>30 \%$ of patients. Besides diagnostic imaging, several biomarkers have proven to be clinically useful in discriminating $\mathrm{AAD}$ from its mimicking conditions, including C-reactive protein (CRP), matrix metalloproteinases (MMPs), soluble elastin fragments (sELAF), D-dimer (a fibrin degradation product), smooth muscle myosin heavy chain, calponin, $\mathrm{N}$-terminal pro brain natriuretic peptide (NT-proBNP), and endothelin precursor [Nienaber 2016; Segreto 2014]. Most interestingly, recent large cohort studies showed that when a risk score $(0-1)$ and D-dimer or soluble isoform of ST2 (a member of the interleukin-1 receptor family) were present together, AAD could be accurately ruled out without aortic imaging [Suzuki 2018]. However, early diagnosis and biomarker-guided treatments still present a conundrum.

Recent studies on animal models have improved our understanding of the pathological mechanisms of AAD. Loss-offunction research revealed that deficiency of the AAD-related genes SMAD3 (SMAD family member 3) and FBN1 (fibrillin 1) can lead to the loss of structural integrity of the aortic walls [Isselbacher 2016]. Cytokine cascades and their derived inflammatory cells also participate in the onset of aortic dissection [Son 2015]. Although there has been much progress, the exact mechanisms of $\mathrm{AAD}$ have remained elusive.

Due to the complexity of AAD and our limited understanding of its molecular mechanisms, hypothesis-driven discovery strategies of individual biomarkers are always laborious and less effective. For this reason, recently, high-throughput systems biology technologies have been used to globally investigate the molecular backgrounds of $\mathrm{AAD}$, which can greatly facilitate comprehensive screening of biomarkers, and improve our understanding of the underlying mechanisms.

In this review, we systemically describe the currently available biomarkers from the genomic, epigenetic, transcriptomic, proteomic and metabolomic profiles of AAD. We discuss how these systems biology approach-based findings provide novel insights into the pathogenesis and offer new valuable biomarkers to overcome the present diagnostic and therapeutic limitations.

\section{GENOMICS}

About $20 \%$ of AAD cases, especially younger patients, have a genetic or familial predisposition, usually in the form of autosomal dominant diseases. Over the past decades, 
genetic testing has become available for AAD's more frequent syndromes. Among them, Marfan syndrome, which is caused by an FBN1 mutation and accounts for 5\% of AAD patients, has been the most studied [Pyeritz 2017]. Currently, the confirmed genes associated with a high-risk of AAD include FBN1, EFEMP2 (EGF containing fibulin extracellular matrix protein 2), TGFB1 (transforming growth factor beta 1), TGFBR1 (transforming growth factor beta receptor 1), TGFBR2 (transforming growth factor beta receptor 2), MYH11 (myosin heavy chain 11), ACTA2 (actin, alpha 2, smooth muscle, aorta), COL3A1 (collagen type III alpha 1 chain), SLC2A10 (solute carrier family 2 member 10), SMAD3, LOX (lysyl oxidase), FOXE3 (forkhead box E3), MFAP5 (microfibril associated protein 5), MAT2A (methionine adenosyltransferase 2A) and PRKG1 (protein kinase, cGMP-dependent, type I) [Brownstein 2017]. These genetic contributions to the pathogenesis of AAD may be due to their possible roles in perturbing extracellular matrix signaling cascades, and smooth muscle contractile apparatus.

With the development of next genomic sequencing, particularly whole exome sequencing (WES), the identification of novel AAD-related genes has been greatly accelerated. Regalado et al found that SMAD3 mutations can be detected in $2 \%$ of familial thoracic AAD [Regalado 2011]. Hannuksela et al found that individuals carrying the deletion mutation (c3272_3273del, p.Ser1091*) in myosin light chain kinase (MYLK) have a high risk of presenting with AAD, which led to a premature stop codon and nonsense-mediated decay [Hannuksela 2016].

Except for causative genes, some studies also indicate that the majority of AAD subjects do not have evidence of mutations in single genes as a risk factor, but rather have the other susceptibility loci. Two genome-wide association studies (GWAS) have identified a susceptibility locus at $15 \mathrm{q} 21.1$ spanning FBN1, LRP1 (LDL receptor related protein 1), rs11172113, and ULK4 (unc-51 like kinase 4) rs2272007 associated with AAD without syndromic features or a family history [Guo 2016; LeMaire 2011]. These data suggest that genomic variants may also contribute to the pathogenesis of $\mathrm{AAD}$ through a different mechanism.

Generally, these novel causative genes and susceptibility loci could be valuable for identifying additional family members at risk for $\mathrm{AAD}$, and improving the gene-based specific management of the carriers.

\section{EPIGENETICS}

Although an accumulating number of genes responsible for $\mathrm{AAD}$ are validated, it is to be assumed that epigenetic factors may play an important role in gene-environment interactions, thus determining the severity of the phenotypes in AAD.

Currently, only one research study focuses on the epigenetic profiling of AAD. In a recent study, Pan et al revealed that non-CpG methylation was significant in the normal ascending aorta, but there was a loss of methylation in the site of aortic dissection [Pan 2017]. Smooth muscle cells represent one of the major cell types in the aorta. Integrative analysis of DNA methylation and mRNA expression indicated down-regulation of smooth muscle differentiation factors and its targets, suggesting a significant dedifferentiated phenotype in AAD. Furthermore, changes in the epigenome may also mediate the inflammatory vascular remodeling process in response to environmental risk factors such as smoking [Pan 2017]. Therefore, this epigenetic study implies that these phenotype alterations underpinning the loss of non-CpG methylation in $\mathrm{AAD}$ are closely associated with impaired strength of the aortic wall. However, efforts to discover the role of DNA methylation alterations in AAD are still limited, and deserve further investigation in the future.

Different from DNA methylation, epigenetic-related microRNA profiles have been widely investigated in AAD. A series of circulating microRNAs that can be developed as potential diagnostic biomarkers for $\mathrm{AAD}$ have been have identified and subsequently validated, such as miR-4787, miR-4306, miR-15a, miR-23a, miR-25, miR-29a miR-155, and miR-26b [Dong 2017; Wang 2017b; Xu 2017]. Functionally, some AAD-related miRNAs may be involved in the pathogenesis of AAD. For example, miR-4787 and miR-4306 can directly target polycystin 1 (PKD1) and TGF- $\beta 1$, while inflammatory factors TNF- $\alpha$ and TGF- $\beta 1$ can also stimulate the increase of miR-21-5p expression in aortic vascular smooth muscle cells [Kimura 2017]. Besides the blood level, microRNA comparative analysis was also performed on the tissue level, and 30 differentially-expressed microRNAs involved in extracellular matrix (ECM) metabolism, cytoskeleton organization, and inflammation were identified [Wang 2015]. Among them, miR-4313, miR-933, miR-1281, and miR-1238 were differentially expressed in both aortic tissue and plasma in AAD patients [Wang 2015].

\section{TRANSCRIPTOMICS}

The gene expression pattern in $\mathrm{AAD}$-associated cells or tissues can broadly reflect their aberrant functional states and the underlying pathological mechanisms. Currently, most of the transcriptome-based studies on AAD have been performed using microarray platforms to characterize the gene expression profiles of dissected aorta tissues, compared with those from multi-organ donor controls or predisposing diseases such as Marfan syndrome [Kimura 2017; Wang 2017; Pan 2014; Wang 2012; Mohamed 2009; Weis-Müller 2006]. Furthermore, most of these studies focus on Stanford type A $\mathrm{AAD}$; only one research study has used tissues from Stanford type B AAD patients as the starting materials.

Not surprisingly, although hundreds of significant genes or probes have been identified in each transcriptome study, there is little overlap between different datasets, which may be due to the heterogeneity of the samples and platforms used. However, the gene modules responsible for the integrity and strength of the aortic wall are significantly enriched in different studies. Genes encoding for the extracellular matrix components, cell/matrix adhesion, and cytoskeleton (such as collagen, TIMP3 [TIMP metallopeptidase inhibitor 3], TIMP4 [TIMP metallopeptidase inhibitor 3], and SERPINE1 [serpin family E member 1]) are frequently downregulated in aortic dissection, whereas the levels of genes 
promoting matrix degradation (such as MMPs) are always increased. As expected, some inflammatory cytokine genes (such as IL-2, IL-6 IL-8, PDGFA, TGFB1, and VEGFA) are also up-regulated in dissection, which reflects the fact that local inflammation exerts multiple important effects on the destruction of the media layer of the aortic wall in AAD [Weis-Müller 2006]. FKBP11 is another potential target identified from AAD gene expression profiling, which elicits a pro-inflammatory that functions in facilitating the colonization of circulating monocytes into the aorta, thus activating macrophages to degrade proteins [Wang 2017].

Transcriptome data can also indirectly reflect the status of pathway activation during AAD development. Kimura et al found that TNF- $\alpha$ and TGF- $\beta$ might be upstream cytokines affecting the gene expression profiles of the dissected aorta [Kimura 2017]. More interestingly, two studies independently confirmed Janus kinase 2 (JAK2), a kinase regulating inflammatory response, as a centered hub gene in the network modules related to AAD. This indicates that JAK2 may contribute to the development of AAD [Kimura 2017, Pan 2014]. However, a recent study indicated that JAK2 may exert a protective effect on aortic dissection associated acute lung injury. Therefore, the exact role of JAK2 on AAD should be further evaluated [Ren 2017].

The available transcriptomic changes strongly support the idea that $\mathrm{AAD}$ is mechanistically related to cytokine mediated inflammatory pathway activation, and the subsequent decreased expression of genes responsible for the integrity and strength of the aortic walls.

\section{PROTEOMICS}

Mass spectrometry or protein array based proteomic technologies allow for the rapid and accurate detection of hundreds to thousands of proteins isolated from clinical samples. More meaningfully, this approach would also reveal the broad range of posttranslational modifications of specific proteins, which makes it possible to interpret the pathological mechanisms of diseases, with new insights.

Recent studies have shown promising results of proteomic profiling on AAD-related tissues. Schachner et al used the differential in-gel electrophoresis (DIGE) approach to compare the difference in protein expression profiles in ascending aortic wall specimens of AAD, aneurysm, and normal controls [Schachner 2010]. This study identified and validated that alpha-1-antitrypsin (A1AT) was significantly reduced in dissected aortic tissues, but not in those of aneurysms. However, in another iTRAQ-based proteomic study, alpha-1-antitrypsin expression was significantly increased in aortic tissue samples from thoracic aortic dissection with hypertension [Zhang 2015]. This discrepancy can be attributed to the existing different isoforms of alpha-1-antitrypsin, but the exact role of A1AT in AAD development still needs further investigation. Except for A1AT, the latter study also confirmed that fibrillin-1, emilin1 , decorin, protein DJ-1 and histone $\mathrm{H} 4$ were dysregulated in AAD [Zhang 2015]. Furthermore, similar to the transcriptomic findings, the proteomic profiling also indicated some function modules, including cell-matrix interactions, ECM remodeling, and IL-6 signaling enriched in the main protein interaction networks related to AAD [Zhang 2015].

Two consecutive studies investigated the serum proteomic variation associated with $\mathrm{AAD}$, using isobaric tags for relative and absolute quantitation to explore new biomarkers that can increase the diagnostic power of traditional methods [Xiao 2016; Gu 2011]. In these studies, lumican, an ECM protein produced by smooth aortic muscle cells, was revealed to be significantly increased in AAD serum, and its levels also correlated with the time from onset to admission for AAD. Furthermore, this novel marker had a specificity of $95 \%$, and the combination of lumican and D-dimer demonstrated superior diagnostic value (sensitivity $88.33 \%$ and specificity $95 \%$ ) [Xiao 2016].

The above protein profiling data provide new insight to understand the development of $\mathrm{AAD}$ and to improve the accuracy of the established diagnostic strategies. However, the current proteomic research on $\mathrm{AAD}$ is still at the beginning. In particular, more efforts are needed to investigate the AAD-associated posttranslational modification changes.

\section{METABOLOMICS}

In many pathological conditions, the content of the metabolites in tissues and blood also fluctuates in response to genetic and environmental factors. Therefore, it is reasonable to speculate that both local and systemic metabolites would change intensively in response to AAD. Recently, several studies began to explore the characteristics of metabolite profiling in AAD.

Using high-performance liquid chromatography-mass spectrometry (HPLC-MS), Ren et al deciphered the variation of small molecule metabolites in the AAD serum. They found 35 that were up-regulated and 105 that were down-regulated only in AAD patients, compared with healthy and hypertension controls [Ren 2017]. These differentially expressed metabolites in $\mathrm{AAD}$ are mainly entrenched in tryptophan, histidine, glycerophospholipid, ether lipid, and choline metabolic pathways. Finally, the authors' proposed combination of the three metabolites AFMK, glycerophosphocholine, and ergothioneine may have potential for AAD screening and auxiliary diagnosis.

Another study belongs to targeted metabolomics and only focuses on the amino acid contents of in the plasma. Eight amino acid contents (histidine, glycine, serine, citrate, ornithine, hydroxyproline, proline, and sarcosine) were significantly different in AAD, compared with coronary heart disease. The two blood metabolites glutamate and phenylalanine were significantly changed in AAD, compared with chronic aortic dissection [Wang 2017a].

Overall, these two proof-of-concept studies support the idea that the use of peripheral blood metabolites showed strong clinical feasibility, and is promising as a complement to the current diagnosis of AAD.

\section{FUTURE PERSPECTIVES}

The gene expression regulation responsible for the development of AAD is complicated and comprehensive. Systems biology platforms provide an unbiased view on the comprehensive gene expression changes in AAD tissues and cells. Multiple cell 
types in aortic walls (smooth muscle cells, fibroblasts, endothelial cells, macrophages, etc.) participate in the pathological development of AAD. Genetic aberration and environmental risk factors such as smoking make the components and cells determining the structural integrity of aortic walls vulnerable to degeneration, and mRNA, microRNA, protein, and metabolite expression patterns also change dramatically in these cells in particular when exposed to inflammatory factors.

The significance of these genomic and functional genomic data on $\mathrm{AAD}$ not only lies in the overlapped genes or modules reproducibly identified across all the platforms, but more importantly, in the the combination of different data sources that can highlight complementary information to deepen our understanding on the pathological mechanisms underlying $\mathrm{AAD}$.

Although the progress in these areas is encouraging, there is still much to do to make better use of the currently available techniques for elucidating novel and important mechanisms for AAD. Unlike the accomplishments in cancer research, most of the current systems biology studies of AAD only focus one or two platforms, and there is still no multi-level data on the same samples available yet. This impedes the mining of novel signaling pathways responsible for AAD development.

Furthermore, until now, although thousands of differently expressed genes, proteins, microRNAs, and metabolites have been identified, only a minor part of them have been confirmed independently. In particular, a few functional validation studies have been performed. Additionally, all the current profiling studies on $\mathrm{AAD}$ used the whole aortic tissues as the starting materials. Considering the complexity of cells involved, it is urgently necessary to carry out system biological studies at the single cell level to more accurately reflecting the changes in each cell types.

\section{CONCLUSION}

Integrating different layers of profiling data can improve our understanding of the underlying mechanisms that cause $\mathrm{AAD}$. Considering the complex nature of $\mathrm{AAD}$, more efforts are needed to decipher the more subtle systematic networks of this multifactorial disease, thus enabling the identification of potential discriminating and drug-able targets for the development of new diagnostic and therapeutic strategies.

\section{REFERENCES}

Brownstein AJ, Ziganshin BA, Kuivaniemi H, et al. 2017. Genes associated with thoracic aortic aneurysm and dissection: An update and clinical implications. Aorta (Stamford) 5:11-20.

Dong J, Bao J, Feng R, et al. 2017. Circulating microRNAs: a novel potential biomarker for diagnosing acute aortic dissection. Sci Rep 7:12784.

Gu G, Cheng W, Yao C, et al. 2011. Quantitative proteomics analysis by isobaric tags for relative and absolute quantitation identified Lumican as a potential marker for acute aortic dissection. J Biomed Biotechnol 2011:920763.

Guo DC, Grove ML, Prakash SK, et al. 2016. Genetic variants in LRP1 and ULK4 are associated with acute aortic dissections. Am J Hum Genet 99:762-9.
Hannuksela M, Stattin EL, Klar J, et al. 2016. A novel variant in MYLK causes thoracic aortic dissections: genotypic and phenotypic description. BMC Med Genet 17:61.

Isselbacher EM, Lino Cardenas CL, Lindsay ME. 2016. Hereditary influence in thoracic aortic aneurysm and dissection. Circulation 133:2516-28.

Kimura N, Futamura K, Arakawa M, et al. 2017. Gene expression profiling of acute type A aortic dissection combined with in vitro assessment. Eur J Cardiothorac Surg 52:810-17.

LeMaire SA, McDonald ML, Guo DC, et al. 2011. Genome-wide association study identifies a susceptibility locus for thoracic aortic aneurysms and aortic dissections spanning FBN1 at 15q21.1. Nat Genet 43:996-1000.

Mohamed SA, Sievers HH, Hanke T, et al. 2009. Pathway analysis of differentially expressed genes in patients with acute aortic dissection. Biomark Insights 4:81-90.

Nienaber CA, Clough RE. 2015. Management of acute aortic dissection. Lancet 385: 800-11.

Nienaber CA, Clough RE, Sakalihasan N, et al. 2016. Aortic dissection. Nat Rev Dis Primers 2:16071.

Pan S, Lai H, Shen Y, et al. 2017. DNA methylome analysis reveals distinct epigenetic patterns of ascending aortic dissection and bicuspid aortic valve. Cardiovasc Res 113:692-704.

Pan S, Wu D, Teschendorff AE, et al. 2014. JAK2-centered interactome hotspot identified by an integrative network algorithm in acute Stanford type A aortic dissection. PLoS One 9:e89406.

Pyeritz RE. 2017. Etiology and pathogenesis of the Marfan syndrome: current understanding. Ann Cardiothorac Surg 6: 595-8.

Regalado ES, Guo DC, Villamizar C, et al. 2011. Exome sequencing identifies SMAD3 mutations as a cause of familial thoracic aortic aneurysm and dissection with intracranial and other arterial aneurysms. Circ Res 109:680-6.

Ren W, Wang Z, Wu Z, et al. 2017. JAK2/STAT3 pathway was associated with the protective effects of IL-22 on aortic dissection with acute lung injury. Dis Markers 2017:1917804.

Ren Y, Tang Q, Liu W, et al. 2017. Serum biomarker identification by mass spectrometry in acute aortic dissection. Cell Physiol Biochem 44:2147-57.

Schachner T, Golderer G, Sarg B, et al. 2010. The amounts of alpha 1 antitrypsin protein are reduced in the vascular wall of the acutely dissected human ascending aorta. Eur J Cardiothorac Surg 37:684-90.

Segreto A, Chiusaroli A, De Salvatore S, et al. 2014. Biomarkers for the diagnosis of aortic dissection. J Card Surg 29:507-11.

Son BK, Sawaki D, Tomida S, et al. 2015. Granulocyte macrophage colony-stimulating factor is required for aortic dissection/intramural haematoma. Nat Commun 6:6994.

Suzuki T, Eagle KA. 2018. Biomarker-assisted diagnosis of acute aortic dissection. Circulation 137:270-2.

Wang L, Liu S, Yang W, et al. 2017. Plasma amino acid profile in patients with aortic dissection. Sci Rep 7:40146.

Wang L, Yao L, Guo D, et al. 2015. Differential expression of microRNAs in aortic tissue and plasma in patients with acute aortic dissection. J Geriatr Cardiol 12: 655-61.

Wang L, Zhang S, Xu Z, et al. 2017. The diagnostic value of microRNA4787-5p and microRNA-4306 in patients with acute aortic dissection. Am J Transl Res 9: 5138-49. 
Wang T, He X, Liu X, et al. 2017. Weighted gene co-expression network analysis identifies FKBP11 as a key regulator in acute aortic dissection through a NF-kB dependent pathway. Front Physiol 8:1010.

Wang Y. 2012. Gene expression profiling in acute Stanford type B aortic dissection. Vasc Endovascular Surg 46:300-9.

Weis-Müller BT, Modlich O, Drobinskaya I, et al. 2006. Gene expression in acute Stanford type A dissection: a comparative microarray study. J Transl Med 4:29.
Xiao Z, Xue Y, Yao C, et al. 2016. Acute aortic dissection biomarkers identified using isobaric tags for relative and absolute quantitation. Biomed Res Int 2016:6421451.

Xu Z, Wang Q, Pan J, et al. 2017. Characterization of serum miRNAs as molecular biomarkers for acute Stanford type A aortic dissection diagnosis. Sci Rep 7:13659.

Zhang K, Pan X, Zheng J, et al. 2015. Comparative tissue proteomics analysis of thoracic aortic dissection with hypertension using the iTRAQ technique. Eur J Cardiothorac Surg 47:431-8. 